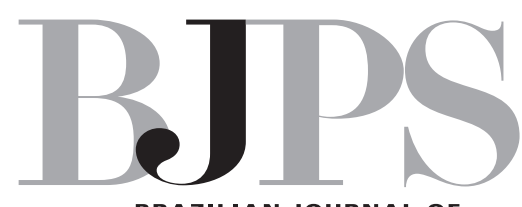

Brazilian Journal of

\title{
Perspectives and challenges in Pharmaceutical Care
}

In recent decades Pharmacy has moved from a product orientation (drug dispensing) to a patient focus. The clinical training requirements for pharmacists have expanded, and besides this process can be considered slow, especially in Latin American countries, it is undeniable that changes are gaining strength. The World Health Organization (WHO), Pan American Health Organization (PAHO), International Federation of Pharmacists (FIP), among others, have organized several meetings, and published important documents in order to transform the concept of Pharmacy from a commodity-based into a clinical profession. This change in focus also implies that the Pharmacy curriculum should be adapted, in order to provide pharmacists with new knowledge and skills. In most countries this change is taking place but not in a very structured manner.

Pharmaceutical Care as a professional practice has been defined in the United States of America in 1990 by Charles Hepler and Linda Strand. From then on, this initiative has become an important practice for pharmacists around the world. Currently, pharmaceutical care is understood as the pharmacists' compromise to obtain the maximum benefit from the pharmacological treatments of the patients, being therefore responsible for monitoring their pharmacotherapy. However, since its introduction there has been a debate on its definition. Probably, the differences in Health Care Systems among the different countries are responsible for this, as well as the problems related to resources, education requirements, and developing skills.

Numerous studies have revealed a relationship between morbidity-mortality and pharmacotherapy. It is known that the control of drug use is necessary, and is mandatory to evaluate the benefits of pharmaceutical care on patients' health. The consequences on society can be measure by the impact of pharmaceutical care provision in common pathologies such as hypertension, diabetes, hyperlipidemia, asthma, rheumatic diseases, psychiatric disorders, and in polymedicated patients. In fact, a large amount of data has being published on clinical, economic and humanistic pharmaceutical care outcomes, and also, on drug therapy problems (DTP) considered as failures in pharmacotherapy.

Not dissimilar from Hepler and Strand's original definition, pharmaceutical care is often regarded in Europe as the process of optimizing the outcome of a patient's drug therapy. The goal of that process is to improve the patient's quality of life (QOL). Van Mil, Schulz, and Tromp (Pharm. World Sci., v. 26, n. 6, p. 303-11, 2004) reviewed the European developments in concepts, implementation, teaching, and research related to pharmaceutical care, which led to the following proposition: "Pharmacists should move from behind 


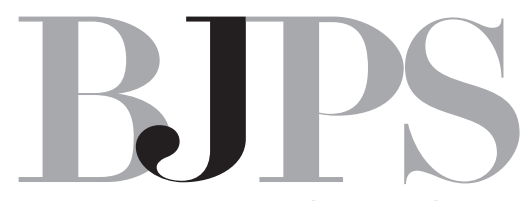

Brazilian Journal of

Pharmaceutical Sciences

the counter and start serving the public by providing care instead of pills only. There is no future in the mere act of dispensing. That activity can and will be taken over by the internet, machines, and/or hardly trained technicians. The fact that pharmacists have an academic training and act as health care professionals puts a burden upon them to better serve the community than they currently do."

In Brazil those issues have been widely discussed by researchers, policy-makers and practitioners. In 2002 the National Council of Education/Ministry of Education and Culture published the Resolution 02 for the implementation of generalist curriculum for Pharmacy courses. In the same year the "Proposal for Consensus on Pharmaceutical Care" was published, which caused the inclusion of pharmaceutical care in the National Agenda of Priorities in Health Research in 2004.

In parallel, it was established the "Research Program to the SUS: Shared Management in Health" (PPSUS). The program is conducted by the Sao Paulo Research Foundation (FAPESP) since 2004 in partnership with the Sao Paulo Health Department (SES-SP) and the National Council for Scientific and Technological Development (CNPq). This initiative also supports the development of research projects that promote education and improvement of health quality in Sao Paulo in the context of the Unified Health System (SUS), which represents a significant contribution to advance of Science, Technology \& Innovation in Health for the implementation of Health Care Networks.

Thereafter there was an increase on pharmaceutical care research in Brazil as showed by FunchalWitzel et al. (Braz. J. Pharm. Sci., v. 47, n. 2, 2011), who evaluated the Brazilian scientific production on this area from 1990 to 2009 by means of a descriptive study of bibliographical basis through selection and analysis of published articles by Brazilians authors in journals indexed in Medline, Embase, Lilacs, Web of Science and International Pharmaceutical Abstracts databases. The results showed that the main researched fields were Community Pharmacy, Hospital Services and Primary Health Care Units. Regarding authors, 72.7 per cent were from the Southeast region, and 62.3 per cent were affiliate with State Universities. The most researched subjects were elderly and patients with chronic diseases, and the authors concluded that: "There is a noticeable increase in the scientific production with regards to pharmaceutical care practice with potential growth in the country, and with practice consolidation, it is expected the increase in number, scope and quality of the research conducted so as to yield knowledge with scientific rigor that contributes for the recognition of the pharmacists actions in the patient-care team."

In this context, the Faculty of Pharmaceutical Sciences at USP, leading research in technological areas related to pharmaceutical profession in Brazil, has made important moves to adequately foster the area of pharmaceutical care, according to the curriculum guidelines of the Ministry of Education and Culture and Health Policies in Brazil, recognizing the need to provide appropriate training for pharmacists in Hospital/ Clinical Pharmacy, Community Pharmacy, and Primary Health Care Units. Regarding pharmaceutical care 
teaching and research, an important decision was taken recently - the transformation of the University Pharmacy at USP (FARMUSP) from a dispensing focused model to a patient-oriented one, supported by projects in partnership with the Sao Paulo Health Department (SES-SP) and the University Hospital of USP (HU-USP).

Finally, it is important to highlight the role of the Brazilian Journal of Pharmaceutical Sciences - BJPS in this scenario. We have observed an increase in submissions of pharmaceutical care scientific articles to BJPS, developed by Brazilian and foreign researchers, which resulted in the publication of 46 articles in the last three years. This fact represents a paradigm shift: although the global pharmaceutical practice is under development in Brazil, there are researchers interested in disseminating the results of their research, which contributes to the essential debate for the consolidation of a new research line.

Furthermore, I believe that Brazil is taking a big step forward by promoting the development of research involving pharmaceutical care. Everyone - patients, health systems, universities, and health care professionals - will be favoured by this advance, since the pharmacist has worked increasingly in multidisciplinary teams.

It is also necessary to emphasize that the role of the university is to promote debate and research in all areas of knowledge, which reminds me two quotes by Johann Wolfgang Von Goethe: "In the process of enlargement of knowledge is occasionally necessary a reorganization. In most cases the reordering takes place by new maximum, but always remains provisional", and "What is worth of looking without seeing?"

Sílvia Storpirtis

Associated Editor 